\title{
Mahalo-Subaru: Mapping Star Formation at the Peak Epoch of Massive Galaxy Formation
}

\author{
Tadayuki Kodama ${ }^{1,2,5}$, Masao Hayashi ${ }^{2}$, Yusei Koyama ${ }^{3,2}$, Ken-ichi \\ Tadaki $^{4,2}$, Ichi Tanaka ${ }^{1}$ and Rhythm Shimakawa ${ }^{5}$ \\ ${ }^{1}$ Subaru Telescope, National Astronomical Observatory of Japan, 650 North A'ohoku Place, \\ Hilo, HI 96720, USA \\ email: t.kodama@nao.ac.jp \\ ${ }^{2}$ Optical and Infrared Astronomy Division, National Astronomical Observatory, Mitaka, \\ Tokyo 181-8588, Japan \\ ${ }^{3}$ Department of Physics, Durham University, South Road, Durham DH1 3LE, UK \\ ${ }^{4}$ Dept. of Astronomy, Graduate School of Science, Univ. of Tokyo, Tokyo 113-0033, Japan \\ ${ }^{5}$ Dept. of Astronomical Science, Graduate University for Advanced Studies, Mitaka, Tokyo \\ 181-8588, Japan
}

\begin{abstract}
MAHALO-Subaru (MApping HAlpha and Lines of Oxygen with Subaru) is our ongoing large programme which aims to investigate how the star forming activities in galaxies are propagated as a function of time, mass, and environment. We are targeting 10 clusters and proto-clusters at $0.4<z<2.6$, and two general fields (GOODS-N and SXDF-CANDELS) with Suprime-Cam and MOIRCS by utilizing our unique sets of narrow-band filters. The narrow-band imaging can map out star forming galaxies with the redshifted Halpha and/or [OII] emission lines from our targets, and thus providing relatively unbiased views of star forming activities across time and environment. We have almost completed narrow-band imaging of our targets, and found that star forming activity is very high even in the proto-cluster cores $(z \gtrsim 1.5)$, and that the peak of star formation is shifted outwards with time, indicating the inside-out formation of clusters. Moreover, we have identified many "red" emitters especially in high density regions at $z>2$, which suggests that the mode of star formation and/or the activation of AGN are dependent on environment, and thus holding the key to the environmental effects at the early stage of cluster galaxies formation and evolution.
\end{abstract}

Keywords. galaxies: clusters, galaxies: evolution, galaxies: formation

\section{Introduction}

The tightness of the colour-magnitude relation of cluster early-type galaxies up to $z \sim 1-1.5$ indicates that star formation in these systems mostly took place long time ago ( $z>1.5-2)$ (e.g., Kodama et al. 1998). Recent near-infrared imaging of proto-clusters suggests that the massive-end of the red-sequence starts to break down finally at $z \gtrsim 2$ (Kodama et al. 2007). It is also suggested that the cosmic star formation rate (SFR) density and the AGN number density both show peaks at $z \sim 2$ (e.g., Bouwens et al. 2008; Fan 2006). All these observations clearly indicate that the redshift interval of $1.5<z<2.5$ (cosmic look-back time of $9-11$ Gyrs) is the critical era for galaxy formation and early evolution. It is therefore essential to systematically map out star forming in the Universe over this period and across various environments. Specifically, we want to know how the star formation in high density regions at high redshifts is intrinsically biased (Cen \& Ostriker 1993), how it is affected by galaxy environment, and how the peak activity is shifted outwards to lower density environments with time, and after all what physical processes are involved and responsible for these phenomena. Our previous Subaru observations show that the star formation activity in the cluster core at $z \sim 1.5$ 
Table 1. Specifications of the narrow-band filters

\begin{tabular}{l|lll}
\hline Camera & Filter & $\lambda_{c}$ & FWHM \\
\hline \multirow{5}{*}{ MOIRCS } & NB1190 & $1.189 \mu \mathrm{m}$ & $0.014 \mu \mathrm{m}$ \\
& NB1550 & $1.550 \mu \mathrm{m}$ & $0.018 \mu \mathrm{m}$ \\
& NB1657 & $1.657 \mu \mathrm{m}$ & $0.019 \mu \mathrm{m}$ \\
& NB2071 & $2.069 \mu \mathrm{m}$ & $0.027 \mu \mathrm{m}$ \\
& NB2095 & $2.095 \mu \mathrm{m}$ & $0.025 \mu \mathrm{m}$ \\
& NB2288 & $2.296 \mu \mathrm{m}$ & $0.023 \mu \mathrm{m}$ \\
& NB2315 & $2.313 \mu \mathrm{m}$ & $0.027 \mu \mathrm{m}$ \\
\hline \multirow{3}{*}{ Suprime-Cam } & NA671 & $0.6714 \mu \mathrm{m}$ & $0.0130 \mu \mathrm{m}$ \\
& NB912 & $0.9139 \mu \mathrm{m}$ & $0.0134 \mu \mathrm{m}$ \\
& NB921 & $0.9173 \mu \mathrm{m}$ & $0.0132 \mu \mathrm{m}$ \\
& NB973 & $0.9755 \mu \mathrm{m}$ & $0.020 \mu \mathrm{m}$ \\
\hline
\end{tabular}

is as high as that in the field, and the peak of star formation is shifted outwards of clusters as time progresses (Koyama et al. 2010; Hayashi et al. 2010). We need to go further back in time to explore the early stage of galaxy formation and evolution and its environmental dependence.

\section{Mahalo-Subaru project}

To understand how galaxies form and evolve at the peak epoch, we have been conducting the "Mahalo-Subaru" project (MApping HAlpha and Lines of Oxygen with Subaru), a large part of which was granted as a Subaru open-use intensive program. We are targeting clusters/proto-clusters at $0.4<z<2.53$, and an un-biased general field SXDF/UDS ( $z=2.19$ and 2.53 slices) (Table 2 ). We employ unique sets of narrow-band (NB) filters on the two wide-field cameras, Suprime-Cam (optical; $34^{\prime} \times 27^{\prime}$ ) and MOIRCS (NIR; $7^{\prime} \times 4^{\prime}$ ) (Table 1). Most of the MOIRCS NB filters are designed and manufactured specifically to our targets, and their wavelengths perfectly match the redshift $\mathrm{H} \alpha$ lines from our $z>1.5$ targets. The Suprime-Cam NB filters in the $z^{\prime}$-band are also used for searching for [OII] emitters at $z=1.46-1.62$. The line-of-sight velocity range that fall within the filter FWHM with respect to the cluster center is optimal $( \pm 1000-3000 \mathrm{~km} / \mathrm{s})$.

Using these NB filters, we have successfully identified lots of star-forming emission line galaxies very efficiently which emit nebular emission lines from ionized star-forming regions, in narrow redshift slices associated with the clusters or in the general field. We are sure about their membership by the presence of emission lines and their SEDs. We have almost completed imaging observations under excellent conditions. Exposure times were typically $1-1.5 \mathrm{hrs}$ per broad-band, and 3-4hrs per narrow-band for $z \gtrsim 1.5$ targets.

We have identified well-visible large scale structures traced by the emitters for all of our cluster targets that were analysed so far. For example, the three high redshift clusters, 2215, 0218, and 0332 at $z=1.5-1.6$ are all embedded in extremely large scale structures traced by [OII] emitters which spread over $\sim 20-30 \mathrm{Mpc}$ in co-moving scale. Also at $z \gtrsim 2, \mathrm{H} \alpha$ emitters in the vicinity of and physically associated with the radio galaxies are spatially strongly clustered and showing clumpy and/or filamentary structures (Fig. 1). For example, three dense star-bursting groups of galaxies are clearly identified in the proto-cluster USS1558; one including a radio galaxy, another located 3 arcmin away to the Southwest, and the other located in between the two. This system is therefore a clumpy forming cluster to be merged together in the near future.

We find that some of the $\mathrm{H} \alpha$ emitters in the proto-cluster fields show very red colours of $J-K_{s}>2.3$ (shown by red filled circles/squares), indicating that they are either dusty 
Table 2. The list of our targets. S-Cam stands for Suprime-Cam.

\begin{tabular}{|c|c|c|c|c|c|c|c|c|}
\hline $\begin{array}{c}\text { environ- } \\
\text { ment }\end{array}$ & target & $z$ & line & $\begin{array}{c}\lambda \\
(\mu \mathrm{m})\end{array}$ & camera & NB-filter & $\begin{array}{l}\text { conti- } \\
\text { nuum }\end{array}$ & $\begin{array}{l}\text { status } \\
\text { as of Oct '12 }\end{array}$ \\
\hline $\begin{array}{l}\text { Low- } z \\
\text { cluster }\end{array}$ & \begin{tabular}{|l|} 
CL0024+1652 \\
CL0939+4713 \\
RXJ1716.4+6708
\end{tabular} & $\begin{array}{l}0.40 \\
0.41 \\
0.81\end{array}$ & $\begin{array}{l}\mathrm{H} \alpha \\
\mathrm{H} \alpha \\
\mathrm{H} \alpha \\
{[\mathrm{OII}]}\end{array}$ & $\begin{array}{l}0.916 \\
0.923 \\
1.190 \\
0.676\end{array}$ & $\begin{array}{l}\text { S-Cam } \\
\text { S-Cam } \\
\text { MOIRCS } \\
\text { S-Cam }\end{array}$ & $\begin{array}{c}\text { NB912 } \\
\text { NB921 } \\
\text { NB1190 } \\
\text { NA671 }\end{array}$ & $\begin{array}{l}z^{\prime} \\
z^{\prime} \\
J \\
R\end{array}$ & $\begin{array}{l}\text { Kodama+'04 } \\
\text { Koyama+'11 } \\
\text { Koyama+'10 } \\
\text { observed }\end{array}$ \\
\hline $\begin{array}{l}\text { High- } z \\
\text { cluster }\end{array}$ & \begin{tabular}{|l|} 
XCSJ2215-1738 \\
4C65.22 \\
CL0332-2742 \\
CIGJ0218.3-0510
\end{tabular} & $\begin{array}{l}1.46 \\
1.52 \\
1.61 \\
1.62\end{array}$ & $\begin{array}{l}\mathrm{OHI}] \\
\mathrm{H} \alpha \\
{[\mathrm{OII}]} \\
{[\mathrm{OII}]}\end{array}$ & $\begin{array}{l}0.916 \\
1.651 \\
0.973 \\
0.977\end{array}$ & $\begin{array}{c}\text { S-Cam } \\
\text { MOIRCS } \\
\text { S-Cam } \\
\text { S-Cam }\end{array}$ & $\begin{array}{l}\text { NB912,921 } \\
\text { NB1657 } \\
\text { NB973 } \\
\text { NB973 }\end{array}$ & $\begin{array}{l}z^{\prime} \\
H \\
y \\
y\end{array}$ & $\begin{array}{l}\text { Hayashi+'10,'11 } \\
\text { observed } \\
\text { Hayashi+'13 } \\
\text { Tadaki+'12 }\end{array}$ \\
\hline $\begin{array}{l}\text { Proto- } \\
\text { cluster }\end{array}$ & $\begin{array}{l}\text { PKS1138-262 } \\
\text { 4C23.56 } \\
\text { USS1558-003 }\end{array}$ & $\begin{array}{l}2.16 \\
2.48 \\
2.53\end{array}$ & $\begin{array}{l}\mathrm{H} \alpha \\
\mathrm{H} \alpha \\
\mathrm{H} \alpha\end{array}$ & $\begin{array}{l}2.071 \\
2.286 \\
2.315\end{array}$ & $\begin{array}{l}\text { MOIRCS } \\
\text { MOIRCS } \\
\text { MOIRCS }\end{array}$ & $\begin{array}{l}\text { NB2071 } \\
\text { NB2288 } \\
\text { NB2315 }\end{array}$ & $\begin{array}{l}K_{\mathrm{s}} \\
K_{\mathrm{s}} \\
K_{\mathrm{s}}\end{array}$ & $\begin{array}{l}\text { Koyama+'13 } \\
\text { Tanaka+'11 } \\
\text { Hayashi+'12 }\end{array}$ \\
\hline $\begin{array}{l}\text { General } \\
\text { field }\end{array}$ & $\mid \begin{array}{l}\text { GOODS-N } \\
\left(70 \operatorname{arcmin}^{2}\right) \\
\\
\text { SXDF-CANDELS } \\
\left(92 \operatorname{arcmin}^{2}\right)\end{array}$ & 2.19 & $\begin{array}{c}\mathrm{H} \alpha \\
\mathrm{H} \beta \\
{[\mathrm{OII}]} \\
\mathrm{H} \alpha \\
\mathrm{H} \beta \\
{[\mathrm{OII}]} \\
\mathrm{H} \alpha\end{array}$ & $\begin{array}{l}2.094 \\
1.551 \\
1.189 \\
2.094 \\
1.551 \\
1.189 \\
2.315\end{array}$ & $\begin{array}{l}\text { MOIRCS } \\
\text { MOIRCS } \\
\text { MOIRCS } \\
\text { MOIRCS } \\
\text { MOIRCS } \\
\text { MOIRCS } \\
\text { MOIRCS }\end{array}$ & $\begin{array}{l}\text { NB2095 } \\
\text { NB1550 } \\
\text { NB1190 } \\
\text { NB2095 } \\
\text { NB1550 } \\
\text { NB1190 } \\
\text { NB2315 }\end{array}$ & $\begin{array}{c}K_{\mathrm{s}} \\
H \\
J \\
K \\
H \\
J \\
K_{\mathrm{s}}\end{array}$ & $\begin{array}{l}\text { Tadaki+'11 } \\
\text { not yet } \\
\text { observed } \\
\text { Tadaki+'13 } \\
\text { not yet } \\
\text { not yet } \\
\text { Tadaki+'13 }\end{array}$ \\
\hline
\end{tabular}

star forming galaxies or passively evolving galaxies with AGNs. Very interestingly, those red $\mathrm{H} \alpha$ emitters tend to be confined in the densest regions (Hayashi et al. 2012; Koyama et al. 2013). This suggests that some dramatic events (such as dusty starbursts or AGN feedback) may be occuring in these galaxies in the dense regions due to environmentally driven galaxy-galaxy interactions, for example. In lower redshift clusters (eg., RXJ1716 at $z=0.81$ ), such red emitters avoid the cluster centre and are seen only in the surrounding groups or filaments (Koyama et al. 2011). Since the location of the red emitters always coincide with the environment where we see a sharp transition in colours or star-formation activity, these must be the key populations under the influence of environmental effects. Star formation histories in the two proto-clusters, 1558 and 1138, are intensively discussed in Hayashi et al. (2012) and Koyama et al. (2013). These proto-clusters are very likely the sites where early-type galaxies, which will eventually dominate clusters by the presentday, are just in their formation phase. They hence provide excellent samples of star forming galaxies with which we can investigate the physical mechanisms of biased galaxy formation and its early evolution in the dense environment.

We also target a blank field, SXDF-UDS-CANDELS, in order to address environmental dependence of star forming galaxies at high redshifts. This field is unique as lots of coordinated multi-waelength data are avaialble, e.g., Subaru Suprime-Cam deep imaging

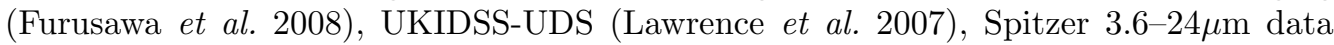
(SpUDS; PI: J. Dunlop), and in particular deep HST high-resolution optical/NIR imaging with ACS/WFC3 (Grogin et al. 2011). Our preliminary analyses show that a large fraction of $\mathrm{H} \alpha$ emitters at $z=2.19$ and 2.53 show clumpy or merger signatures, while others are compact, centrally concentrated objects. They are probably at different stages of galaxy formation with different modes of star formation.

Our Mahalo-Subaru project is providing a unique dataset of star forming galaxies over the peak epoch of galaxy formation and across various environments. It will tell us how 
$\Delta$ R.A. [Mpc (Comoving)]
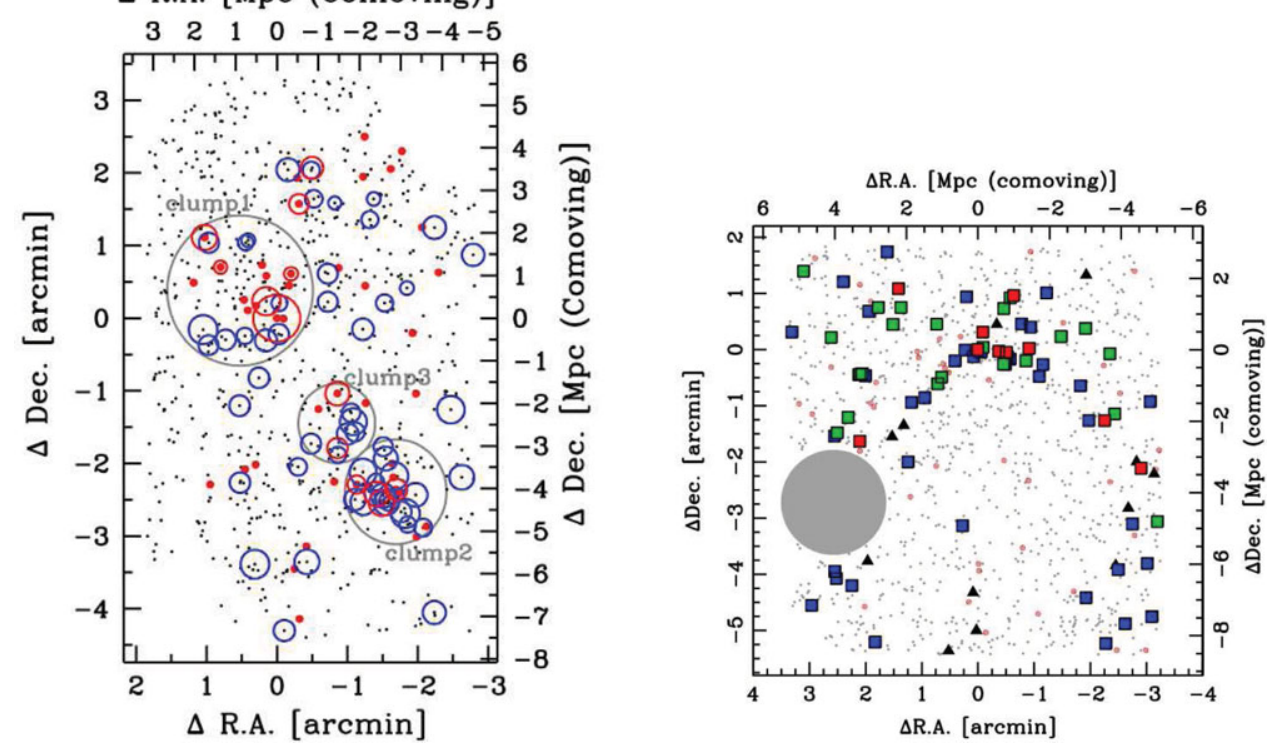

Figure 1. (Left panel): 2-D map of a proto-cluster USS1558-003 at $z=2.53$ (Hayashi et al. 2012). The red and blue open circles indicate $\mathrm{H} \alpha$ emitter candidates associated with the proto-cluster. The size of the circles corresponds to a star formation rate. The filled red circles represent galaxies with $\left(J-K_{\mathrm{S}}\right)>1.38$ (DRGs). See the online color version. (Right panel): 2-D map of a proto-cluster PKS1138-262 at $z=2.16$ (Koyama et al. 2013). The associated H $\alpha$ emitter candidates are shown in colored symbols. The red, green and blue squares indicate $\mathrm{H} \alpha$ emitters with $\left(J-K_{\mathrm{s}}\right)>1.38(\mathrm{DRGs}), 0.8<\left(J-K_{\mathrm{s}}\right)<1.38$ and $\left(J-K_{\mathrm{s}}\right)<0.8$, respectively. The black triangles are the $K_{\mathrm{s}}$-undetected emitters. See the online color version.

galaxy formation has progressed with time and how relevant environmental effects are in shaping present-day massive galaxies.

\section{References}

Bouwens, R. J., Illingworth, G. D., Franx, M., et al. 2008, ApJ, 686, 230

Cen, R. \& Ostriker, J. P. 1993, ApJ, 417, 415

Fan, X. 2006, NewAR, 50, 665

Furusawa, H., Kosugi, G., Akiyama, M., et al. 2008, ApJS, 176, 1

Grogin, N. A., Kocevski, D. D., Faber, S. M., et al. 2011, ApJS, 197, 35

Hayashi, M., Kodama, T., Koyama, Y., et al. 2010, MNRAS, 402, 1980

Hayashi, M., Kodama, T., Koyama, Y., et al. 2011, MNRAS, 415, 2670

Hayashi, M., Kodama, T., Tadaki, K., et al. 2012, ApJ, 757, 15

Lawrence, A., Warren, S. J., \& Almaini, O. 2007, MNRAS, 379, 1599

Kodama, T., Arimoto, N., Barger, A. J., et al. 1998, A\&\&A, 334, 99

Kodama, T., Balogh, M. L., Smail, I., et al. 2004, MNRAS, 354, 1103

Kodama, T., Tanaka, M., Arimoto, N., et al. 2005, PASJ, 57, 309

Kodama, T., Tanaka, I., Kajisawa, M., et al. 2007, MNRAS, 377, 1717

Koyama, Y., Kodama, T., Shimasaku, K., et al. 2010, MNRAS, 403, 1611

Koyama, Y., Kodama, T., Nakata, F., et al. 2011, ApJ, 734, 66

Koyama, Y., Kodama, T., Tadaki, K., et al. 2013, MNRAS, 428, 1551

Tadaki, K., Kodama, T., Koyama, Y., et al. 2011, PASJ, 63, 437

Tadaki, K., Kodama, T., Ota, K., et al. 2012, MNRAS, 423, 2617

Tanaka, I., De Breuck, C., Kurk, J., et al. 2011, PASJ, 63, 415 